\title{
In Some Cases, Once May Be Enough
}

A review of: Clarke G, Collins RA, Leavitt BR, Andrews DF, Hayden MR, Lumsden CJ, McInnes RR 2000 A one-hit model of cell death in inherited neuronal degenerations. Nature 406:195-199

$\mathrm{F}_{\mathrm{a}}$ OR MANY YEARS it has been generally assumed that the rate of cell death within a population of neurons at risk for degeneration increases as the subject ages. This was attributed to a cumulative damage effect, where exposure to events such as oxidative and environmental stresses progressively brings a population of cells toward a critical threshold level from which it cannot recover. As time passes, the likelihood of any given cell in this population crossing that threshold value would increase. A recent study by Clarke et al. challenges this viewpoint, however, and provides compelling data to suggest that the risk of cell death remains constant, and does not increase over time in neuronal populations at risk for degeneration. In this report, Clarke et al. examined the percentage of neurons remaining in at risk populations over time in several neurodegenerative models. If the increasing risk hypothesis were to be correct, then a sigmoidal function would be predicted to best fit the progression of cell loss, as the rate at which cells die would increase progressively over time. Surprisingly, however, none of the 16 different models of neurodegeneration examined were best fit by a sigmoidal decay function. Rather, each was best fit by an exponential decay equation. Such a mathematical model excludes the increased risk model, and supports a model in which the rate of cell loss is either constant, or perhaps decreases, throughout the course of the condition.

\section{JAMES H. EUBANKS}

To account for their results, Clarke et al. propose a model in which individual cells in subjects with a degenerative condition exist in a mutant steady state. Each individual condition would have its own mutant steady state, which would differ from the steady state observed in non-affected individuals. This mutant steady state would reside closer to a critical threshold that when reached, activates degenerative processes within the cell. The more severe the condition, the closer the mutant steady state would reside to this threshold value. Thus, any individual sporadically occurring event that pushes the cell over threshold would lead to its death. This 'one hit' model is equally likely to affect any given cell in a population at any time, and is not influenced by previous events to which the cell may have been exposed.

These observations have far reaching implications. A constant or decreased rate of cell loss throughout the course of a neurodegenerative condition would suggest that clinical treatments given later in time are equally likely to provide benefit to the remaining cells as those given at earlier times. This differs from the cumulative risk model, in which the likelihood of a treatment being efficacious decreases progressively as the cells increase their risk of death. Furthermore, the 'onehit' model suggests that any early in- tervention that moves the mutant steady state away from threshold would provide greater benefit over the course of the lifetime, as the number of spontaneously occurring events reaching threshold would be - in principal - permanently decreased. This is also not necessarily the case for the cumulative hypothesis model, in which if the defect itself were not corrected, the early treatment would likely only slow the progression of the degeneration. And finally, the "one-hit" model suggests that the treatment scheme need not be directly targeted to the molecular cause of the condition. Any intervention that changes the mutant steady state to a level further away from threshold would be beneficial, irrespective of whether it acts on the factor, or system, responsible for the condition. In several experimental model systems, large numbers of somewhat diverse interventions have afforded neuroprotection to at risk neuronal populations. The specific targets of these agents were therefore implicated as playing a role in the degenerative process. The article published by Clarke et al. suggests that alternative interpretation of these results may have to be considered.

Senior Staff Scientist

Division of Cellular and Molecular Biology

Toronto Western Research Institute

University Health Network 